\title{
Preface to Special Issue on Algorithmic Game Theory
}

\author{
Spyros Kontogiannis • Elias Koutsoupias • \\ Pavlos Spirakis
}

Published online: 7 April 2013

(C) Springer Science+Business Media New York 2013

This special issue of TOCS is devoted to the third edition of the International Symposium on Algorithmic Game Theory (SAGT2010). SAGT is an interdisciplinary scientific event that provides a forum for researchers and practitioners to exchange ideas and share new research results. The third edition of SAGT was organized by the Computer Technology Institute and Press "Diophantus" and the University of Athens and took place in Athens on October 18-20, 2010.

The volume contains six papers that derive from and extend the best papers that were accepted and presented in SAGT2010, according to the ranking during the reviewing process. All of the invited contributions have been refereed in accordance with the journal's customary high standards. In a nutshell, the volume contains the following contributions:

- Auletta et al. study the performance of noisy best-response (a.k.a. logit) dynamics, by means of expected social welfare at stationarity and rate of convergence of the underlying process, by providing almost tight bounds for particular classes of games.

- Brandt et al. study another well-known dynamics for strategic games, the fictitious play. The paper shows that the rate of convergence of fictitious play (although

\footnotetext{
S. Kontogiannis $(\bowtie)$

Dept. of Computer Science, University of Ioannina, 45110 Ioannina, Greece

e-mail: kontog@cs.uoi.gr

E. Koutsoupias

University of Athens, Panepistimiopolis, Ilissia, 15784 Athens, Greece

e-mail: elias@di.uoa.gr

P. Spirakis

Computer Engineering and Informatics Department, University of Patras, 26500 Patras, Greece

e-mail: spirakis@cti.gr
} 
it converges) may require an exponential number of rounds, even for symmetric constant-sum win-lose-tie games.

- Mihalák and Schlegel significantly contribute to bounding the structure of equilibria and the price of anarchy in network creation games, for the SumGame and the MaxGame variants (differentiating in the way of determining usage-costs), by proving a tree structure of the equilibria and constant price of anarchy, in almost all cases.

- Anshelevich et al. prove the existence and demonstrate an efficient construction of partition equilibria in resource selection games. The constructed partition equilibrium is also a Nash equilibrium, implying that one does not need to sacrifice the stability of individual players when forming solutions stable against coalitional deviations.

- Macko et al. demonstrate the fundamental differences of Braess's paradox in the context of congestion games with flows over time, compared to the case of games with static flows. It is shown that there are networks which do not admit Braess's paradox in the static case, which admit it in the model with flow over time. Even the severity Braess's ratio may be much different in games with flows over time.

- Fabrikant et al. study the class of weakly acyclic games, in which natural distributed dynamics, such as better-response dynamics, cannot enter inescapable oscillations. The authors show that the existence of a unique pure Nash equilibrium in every subgame implies the weak acyclicity of a game. In contrast, the possible existence of multiple pure Nash equilibria in every subgame is insufficient for weak acyclicity in general. 